\title{
PRÉFACE DE L'AUTEUR.
}

En publiant ces démonstrations cliniques des maladies congéniales et acquises de l'oil humain et de ses amnexes, nous nous proposons de combler une lacume, qui n'est pas moins sensible pour celui qui étudie l'ophthalmologic que pour celui qui l'enseigne.

La science possède un grand nombre d'ouvrages contenant de bonnes et fidèles images des maladies oculaires, mais c'est en vain que nous y cherchons un tableau systématique représentant leur forme extérieure à côté des documents anatomiques relatifs à leur siége et à leur nature. Pour remédicr à cc besoin scientifique de notre époque, nous avons réuni dans un ensemble systématique un grand nombre de figures dessinées sous nos yeux d'après nature, et et nous y avons ajouté des explications en rapport avec le sujet.

Les matériaux que nous allons offrir à nos lecteurs forment une collection choisie des transformations morbides des tissus de l'ueil ; car nous avons jugé à propos, afin de ne pas nous ćtendre outre mesure, d'exclure de nos cadres les phénomènes pathologiques, éphémères et fugaces qui n’aboutissent à aucune altération anatomique. Quant à la classification de ees matériaux, nous avons adopté la marche suivie pour l'anatomic physiologique ; cette marche nous ayant paru plus commode pour établir l'ordre au milieu de tant d'observations et de recherches détaillées. Ainsi, nous commençons dans notre exposition par les altérations de la conjonctive, et nous procédons d'après l'ordre anatomique à la démonstration des maladies des organes plus profonds de l'oeil. Tout autre système conduirait infailliblement à la séparation artificielle des états morbides que l'on observe réunis, et à la réunion de ceux qui sont séfarés dans la nature; tandis que la méthode que: nous avons choisie fait de chaque planche un groupe à part pourvu d'une descrip-tion qui constitue, dans le texte en regard, un chapitre séparé. - Nous espérons que cette méthode sera plus avantageuse que toute autre à ceux qui se serviront de notre ouvrage, et qu'elle répondra aussi bien aux besoins des élèves qu'à celui des professeurs. Puisse ce traité rectifier daus l'esprit des uns et des autres les fiusses opinions sur lat nature et la pathogénie de plusicurs états morbides de l'weil! - Puisse-1-il étenfíre des hypothèses par la seule antorité de faits qu’il contient! En 
favorisant l'étude de l'ophthalmologie, peut-être deviendra-t-il, un jour, le centre autour duquel se grouperont les travaux futurs des savants.

Un traité des maladies de l'œil et de ses annexes, que nous nous proposons de publier dans peu de temps, nous fournira l'occasion d'éclaircir plusieurs faits renfermés dans cet ouvrage, et de porter un jugement sérieux sur leur valeur. - Nous recommandons d'avance ce traité, comme étant le commentaire de l'œuvre que nous soumettons aujourd'hui à la publicité : il poụrra, s'il est bien reçu par le monde savant, se compléter de temps en temps par des cahiers supplémentaires.

A ces quelques mots ajoutons encore une notice sur l'histoire de cet ouvrage , car il a déjà son histoire. Il y a environ deux ans que j'en ai conçu la première idće. Médecin du grand établissement des aveugles à Dresde, ainsi que de la société de charité pour le traitement des maladies des yeux, placé à la tête d'une nombreuse clientèle oculistique, j'ai pu facilement m'entourer de matériaux nombreux pour réaliser mon projet. Je vois encore devant moi les premiers dessins faits d’après nature. - Comme ils répondirent mal à mon désir! Gombien de difficultés n'a-t-il pas fallu surmonter depuis ce moment pour trourer des artistes, pour stimuler leur zèle à reproduire fidèlement la nature, pour développer leurs talents dans cette direction! etc., ete. - Je les ai trouvés enfin, je les ai formés conformément à mon but. Que M. Maurice Kranz, le brave artiste, et M. Hettenhausser, le peintre distingué, veuillent bien recevoir ici mes remercîments. - Mais aussi combien n'a-t-il pas fallu vaincre de difficultés et faire d'instances, pour déterminer les malades à se laisser dessiner par eux! - Combien de fois n'a-t-il pas été nécessaire de recourir à des subterfuges pour ne pas laisser échapper l'occasion favorable d'enrichir la science, surtout quand il a étó urgent de modeler après la mort, ou de recourir à des autopsies! Quels soins et quelle exactitude ne fillait il pas pour obtenir des images fidèles des préparations anatomiques que le moindre retard pouvait endommager, et que leur conservation dans l'esprit-de-vin aurait pu détériorer! Combien de temps a été nécessaire pour achever li collection ct compléter tous les détails! quelle persévérance a-t-il fallu opposer aux diflicultés! Certainement je n'aurais pas enrie de recommeneer un si pénible travail une deuxième fois.

Mais qu'est-il arrivé plus tard? - L'ouvrage était terminé, n'attendait plus que la dernière main pour être achevé ; mais il n'y avait pas d'éditeur pour le livrer à la publicité. J'étais loin d'être aussi heureux que Demours, qui avait pu publier son précieux ouvrage aux frais du gouvernement. Plusieurs maisons bien connues en Allemagne ayant refusé leur concours dans une entreprise évidemment plus avantagreuse à la science que luerative pour le commerce, j'eus l'idée de m'adresser cu Angleterre, lorsque, gràce à l'intervention amieale de M. Dieffenbach de Berlin, M. Reimer sc chargea de la publication. Cet homme honorable, qui seutint me laborieux et pénibles eftorts, mutrepris diuns l'intért de la scionee et de l'lumanite, 
trouvera dans sa conscience la récompense de son désintéressement; il a assuré à l'Allemagne la propriété d'un ouvrage qui certainement pourra soutenir sa réputation de patiente activité et la gloire de ses artistes.

Avant de terminer cette préface, il me reste encore un devoir à remplir, -. c'est celui de la. reconnaissance envers mes honorables collègues, les docteurs $S c i$ ler, Choulant, Hedenus, Schray, Kuhn, Franzel, M. Jieger, Gescheidt, Meding, Arnold, Zeis, Schoen, de Rosas, Beger, Warnatz, Guinther, Behr, Sommering et Martini. - C'est à leur bienveillante sympathic que j’ai dû souvent l'occasion d'examiner des cas rares et de disséquer des yeux malades. Que M. le professeur Ficher de Prague veuille surtout recevoir mes sincères remerciments pour l'envoi d'une collection de cristallins cataractés qu'il avait extraits sur l'œil vivant.

Dresde, octobre 1857. 
